\title{
Tunability of Photonic Band Gaps in One- and Two-dimensional Photonic Crystals Based on ZnS Particles Embedded in $\mathrm{TiO}_{2}$ Matrix
}

\author{
Amel LABBANI and Abdelmadjid BENGHALIA \\ Microwave and Semiconductors Laboratory, Faculty of Engineering Sciences, MENTOURI University Constantine, \\ Ain El Bey Road, 25000 Constantine, Algeria \\ *Corresponding author: Amel LABBANIＥ-mail: Labbame@yahoo.fr
}

\begin{abstract}
Using the Maxwell-Garnett theory, the evolution of the refractive index of titanium dioxide $\left(\mathrm{TiO}_{2}\right)$ doped with zinc sulfide $(\mathrm{ZnS})$ particles is presented. The presence of the nano-objects in the host matrix allows us to obtain a new composite material with tunable optical properties. We find that the filling factor of $\mathrm{ZnS}$ nanoparticles greatly alters photonic band gaps (PBGs). We have calculated also the photonic band structure for electromagnetic waves propagating in a structure consisting of $\mathrm{ZnS}$ rods covered with the air shell layer in $2 \mathrm{D}$ hexagonal and square lattices by the finite difference time domain (FDTD) method. The rods are embedded in the $\mathrm{TiO}_{2}$ background medium with a high dielectric constant. Such photonic lattices present complete photonic band gaps (CPBGs). Our results show that the existence of the air shell layer leads to larger complete photonic gaps. We believe that the present results are significant to increase the possibilities for experimentalists to realize a sizeable and larger $\mathrm{CPBG}$.
\end{abstract}

Keywords: Photonic crystals, photonic band gap, nanoparticles, $\mathrm{ZnS}, \mathrm{TiO}_{2}$

\section{Introduction}

Photonic crystals (PCs) [1, 2] are artificial materials with a periodically modulated dielectric permittivity. They scatter photons in manner similar to the scattering of electrons in the semiconductor $[3,4]$. Light propagation in the PC can be inhibited for a certain frequency range resulting in the creation of the photonic band gap (PBG). The formation of PBGs can be controlled by geometrical parameters such as lattice types and the period, as well as by choosing the constituent materials and their refractive index contrast. One of the most interesting properties of PCs is that they can be designed to possess a complete photonic band gap (CPBG): a frequency region where the light is totally reflected for both polarizations and all directions of the propagation. This may bring about some remarkable physical phenomena such as waveguides, micro-cavities, laser physics, and optical communication. In this paper, we study the optical properties of $\mathrm{TiO}_{2}$ in the presence of $\mathrm{ZnS}$ nanoparticles. Titanium dioxide has attracted much attention in the past as its chemical stability, high refractive index allow it to be used as components in optoelectronic devices, sensors, and photocatalysts [5]. $\mathrm{ZnS}$ with wide and direct band energies of $3.7 \mathrm{eV}$ has attracted much interest of researches because of its excellent properties of luminescence [6] and photochemistry [7]. Its applications in PC devices operating in the visible and near infrared (IR) region due to high refractive indices and large band

Received: 21 October 2011 / Revised version: 15 February 2012

(C) The Author(s) 2012.This article is published with open access at Springerlink.com 
gaps make it highly transparent in the visible region [8]. We have calculated the photonic band gaps in one-dimensional (1D) photonic crystals of alternating layers of the composite material $\left(\mathrm{ZnS}+\mathrm{TiO}_{2}\right)$ on the $\mathrm{TiO}_{2}$ substrate. We have investigated the influence of the volume fraction occupied by $\mathrm{ZnS}$ nanoparticles in the $\mathrm{TiO}_{2}$ matrix on PBGs. 2D core-shell photonic crystals, square and hexagonal lattices for which the effect of the thickness of the shell layer exists between the $\mathrm{ZnS}$ rods and the background on the CPBG are studied also in this paper. Core-shell PCs are composed of core-shell particles (3D) or rods with the cladding (2D) [9-12]. Details of the calculations and discussion of the results will be presented in the remainder of the paper.

\section{Determination of optical index of the composite material using the Maxwell -Garnett theory}

In this section, we will demonstrate that it is possible to modify and tune the optical properties of a $\mathrm{TiO}_{2}$ matrix doped with $\mathrm{ZnS}$ nanoparticles. To define the dielectric permittivity, $\tilde{\varepsilon}(\omega)$, of such a new composite system, $\mathrm{ZnS}+\mathrm{TiO}_{2}$, we use the Maxwell-Garnett theory [13]:

$$
\tilde{\varepsilon}(\omega)=\varepsilon_{m} \frac{\varepsilon_{n}(1+2 f)+2 \varepsilon_{m}(1-f)}{\varepsilon_{n}(1-f)+\varepsilon_{m}(2+f)}
$$

where $\varepsilon_{n}(\omega)$ is the dielectric permittivity of the $\mathrm{ZnS}$ nanoparticle, $\varepsilon_{m}$ is the dielectric permittivity of the $\mathrm{TiO}_{2}$ matrix, and $f$ is the volume fraction occupied by $\mathrm{ZnS}$ nano-objects. We can deduce the real and imaginary parts of $\tilde{\varepsilon}$ by the expression:

$$
\tilde{\varepsilon}(\omega)=\varepsilon^{\prime}(\omega)+\mathrm{i} \varepsilon^{\prime \prime}(\omega) .
$$

The complex refractive index of a composite material can be computed with (3) [14]:

$$
\tilde{n}=n+\mathrm{i} k \text {. }
$$

Equations (2) and (3) are not independent. The relation between $\tilde{n}$ and $\tilde{\varepsilon}$ is

$$
\tilde{n}^{2}=\tilde{\varepsilon}
$$

with real and imaginary parts:

$$
\begin{aligned}
& \varepsilon^{\prime}=n^{2}-k^{2} \\
& \varepsilon^{\prime \prime}=2 n k .
\end{aligned}
$$

We can deduce $n$ and $k$ from (5) and (6) [14], where

$$
\begin{aligned}
& n=\sqrt{\frac{\sqrt{\varepsilon^{\prime 2}+\varepsilon^{\prime \prime 2}}+\varepsilon^{\prime}}{2}} \\
& k=\sqrt{\frac{\sqrt{\varepsilon^{\prime 2}+\varepsilon^{\prime \prime 2}}-\varepsilon^{\prime}}{2}} .
\end{aligned}
$$

Equation (1) can be applied to all types of dielectric matrices containing various kinds of small-particle inclusions [14]. Figure 1 shows a schematic structure of $\mathrm{ZnS}$ nanoparticles distributed randomly in the $\mathrm{TiO}_{2}$ matrix. The fact that the optical index of $\mathrm{ZnS}$ has nonzero imaginary part in the visible region implies that the material will absorb the light passing through its region. However, $\mathrm{TiO}_{2}$ remains transparent at the wavelength investigated.

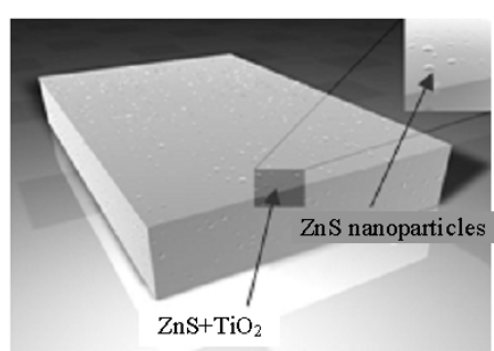

Fig. 1 Schematic structure of $\mathrm{TiO}_{2}$ matrix containing $\mathrm{ZnS}$ nanoparticles.

To describe the optical properties of the new composite material ( $\mathrm{ZnS}$ grains in the $\mathrm{TiO}_{2}$ matrix) with different filling factors $f$, we use the Maxwell-Garnett (MG) theory [13]. The results are shown in Figs. 2(a) and 2(b). These results clearly show that the filling factor has an obvious influence on the real and imaginary parts of the optical index of the MG composite. We can have a decrease of $7.25 \%$ for the real part of $\tilde{n}$ when $f$ ranges from $25 \%$ 
to $65 \%$ at the wavelength of $0.6199 \mu \mathrm{m}$, shown in Fig. 2(a).

Firgure 2(b) shows absorption spectra in the visible range, and these, as can be observed, are more prominent when $f$ increases. These absorption bands are the $\mathrm{ZnS}$ nano-objects signatures in the $\mathrm{TiO}_{2}$ matrix.

It means that the nanocomposite material has $\mathrm{ZnS}$ optical properties in this domain.

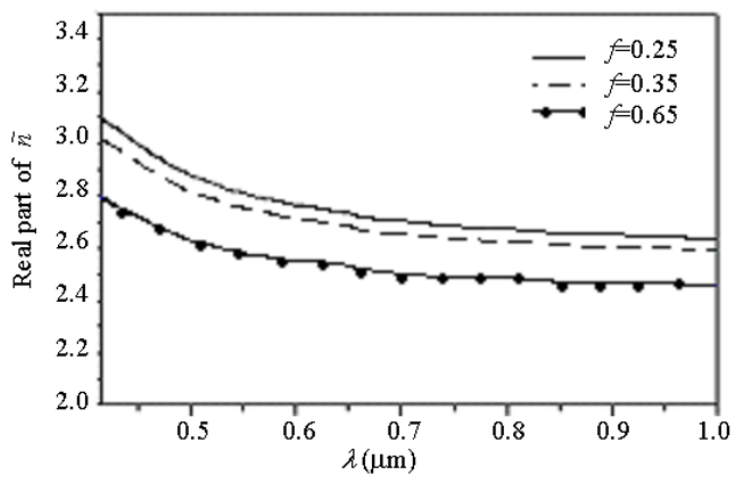

(a)

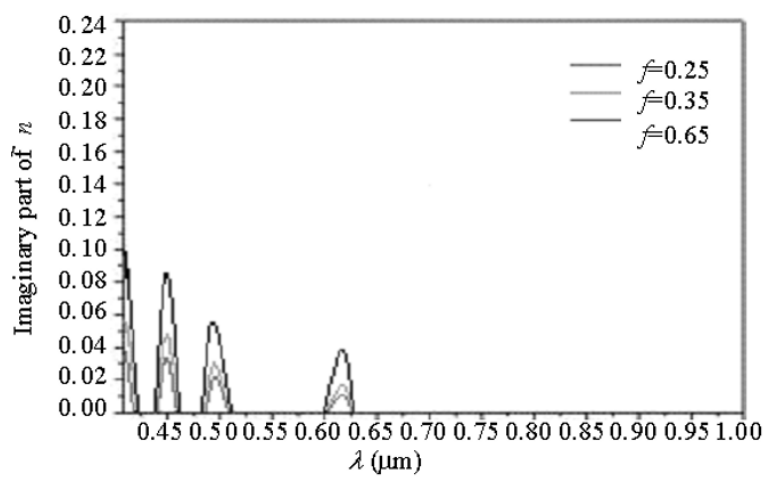

(b)

Fig. 2 Modification of the (a) real part and (b) imaginary part of the composite optical index $\tilde{n}$ of the $\mathrm{TiO}_{2}$ matrix doped with $\mathrm{ZnS}$ particles.

\section{1D photonic band modulation effects due to the nanoparticles concentration}

We model the $\mathrm{ZnS}$ nanoparticles in the $\mathrm{TiO}_{2}$ matrix as a layer with an effective dielectric permittivity $\tilde{\varepsilon}$ obtained by the Maxwell-Garnett formula [13]. To illustrate the influence of the nanoparticle concentration on the PBG, we have calculated band structures in one-dimensional PC of alternating layers of the $\mathrm{ZnS}+\mathrm{TiO}_{2}$ and $\mathrm{TiO}_{2}$ on the
$\mathrm{TiO}_{2}$ substrate as schematically shown in Fig. 3. This periodic structure can be realized by the phase mask or an interferogram, as has been done with $\mathrm{Ag}$ particles in the gelatine [15]. The thickness of the considered layers of the host matrix and the composite material are $50 \mathrm{~nm}$ and $60 \mathrm{~nm}$, respectively. The surrounding medium has a dielectric permittivity $\varepsilon_{m}=8.585$ [16] at the wavelength $(\lambda)$ of $575 \mu \mathrm{m}$. We consider only normal incidence of the electromagnetic wave on the PC. Figures 4(a) and 4(b) show the PBGs for $f=65 \%$ and

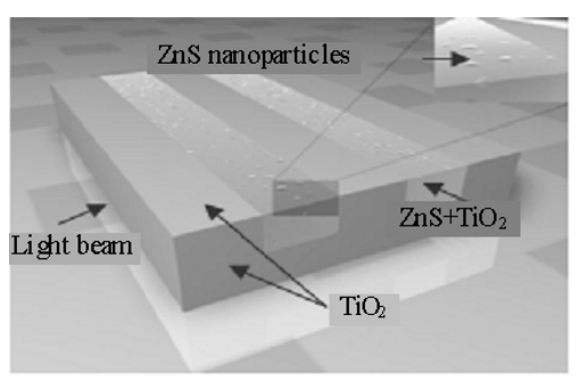

Fig. 3 Schematic structure of the Bragg mirror $\mathrm{ZnS}+\mathrm{TiO}_{2}$ $\mathrm{TiO}_{2}$.

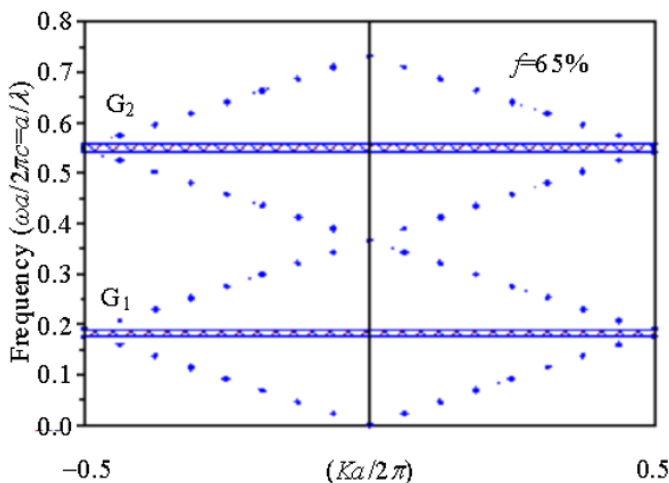

(a)

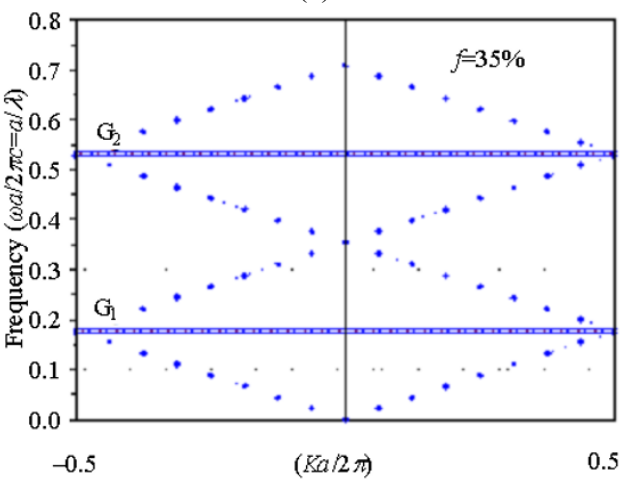

(b)

Fig. 4 Photonic band structure of 1D PC consisting of $\mathrm{ZnS}+\mathrm{TiO}_{2}$ and $\mathrm{TiO}_{2}$ layers for (a) $f=65 \%$ and (b) $f=35 \%$. 
$f=35 \%$, respectively. Results are presented in terms of frequencies $\omega a / 2 \pi c=a / \lambda$, where $a$ is the lattice constant, $c$ is the speed of the light in a vacuum, and $\lambda$ is the vacuum wavelength. From the Fig. 4(a), one may observe 2 CPBGs in the frequency ranges of $(0.1754-0.1908) \omega a / 2 \pi c$ for the first gap $G_{1}$ and $(0.5416-0.5567) \omega a / 2 \pi c$ for the 2 nd gap $\mathrm{G}_{2}$.

\section{2D photonic band modulation effects due to the thickness of the shell layer}

To analyze the variation in the size of the CPBG with the various thicknesses of the interfacial layer, we consider the following two structures:

(1) PC composed of the hexagonal lattice of circular $\mathrm{ZnS}$ rods surrounded by the air in the $\mathrm{TiO}_{2}$ matrix as shown in Fig. 5(a).

(2) PC composed of the square lattice of circular $\mathrm{ZnS}$ rods surrounded by the air in the $\mathrm{TiO}_{2}$ matrix as shown in Fig. 5(b).

Figure 5(c) shows the diagrammatic presentation of the regular core-shell rod. Parameters $r_{c}$ and $r_{s}$ denote the radius of the internal rod and the external of the shell layer. The thickness of the shell layer is then $t_{s}=r_{s}-r_{c}$.

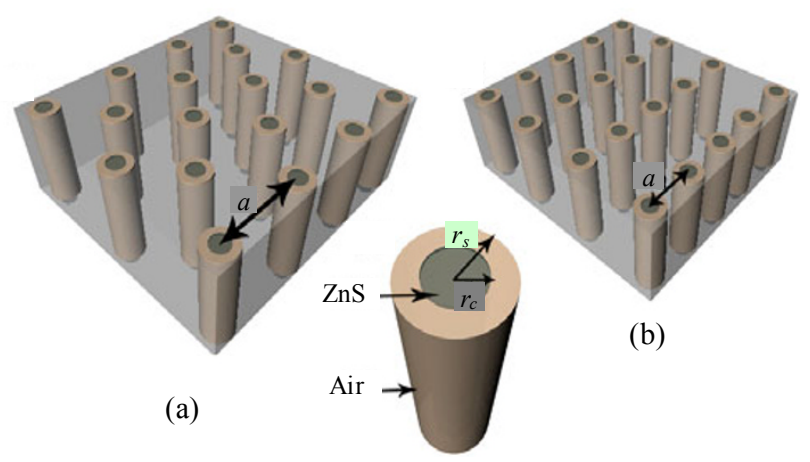

(c)

Fig. 5 Schematic representation of the $2 \mathrm{D}$ core-shell photonic crystal: (a) the hexagonal lattice, (b) the square lattice, and (c) the diagrammatic presentation of a regular core-shell rod.

\subsection{Hexagonal lattice}

Firstly, the analysis has been done for the photonic crystal structure composed of the hexagonal lattice of $\mathrm{ZnS}$ rods surrounded by the air layer as shown in Fig. 5(a). Parameter $a$ is the space between the centers of two nearest-neighbour rods. The dielectric permittivities of the inner rod and the outer shell-layer are fixed to be $\varepsilon_{c}=5.64$ [16] and $\varepsilon_{s}=1$, respectively, at the wavelength of $575 \mu \mathrm{m}$. The permittivity of the surrounding medium $\varepsilon_{m}=8.58$ [16] is greater than that of the shell layer.

The photonic bands of 2D PCs are calculated using the finite difference time domain (FDTD) [17].

The bands for transverse-electric (TE) and transverse-magnetic (TM) polarization modes are traced along the $\Gamma-\mathrm{M}-\mathrm{K}-\Gamma$ path edge for the Brillouin zone.

We can see from Figs. 6(a) and 6(b) that there is only one $\mathrm{CPBG}$ in the frequency range, originating from the overlap of $H_{1-2}$ and $E_{2-3}$ gaps. We denote the gap by $E_{n}$ (respectively $H_{n}$ ) occurring between the $n$-th and the $(n+1)$-th bands of $E$ (respectively $H$ ) polarization.

Figure 6(a) shows the photonic band structure for

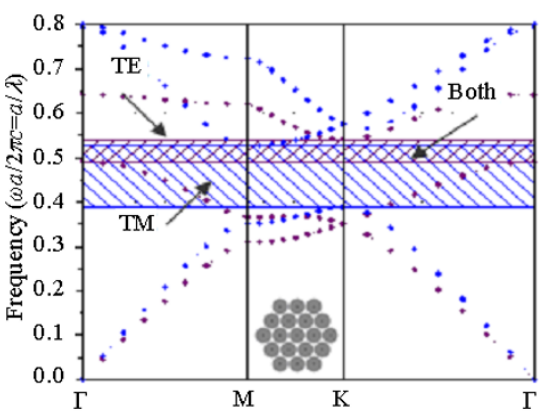

(a)

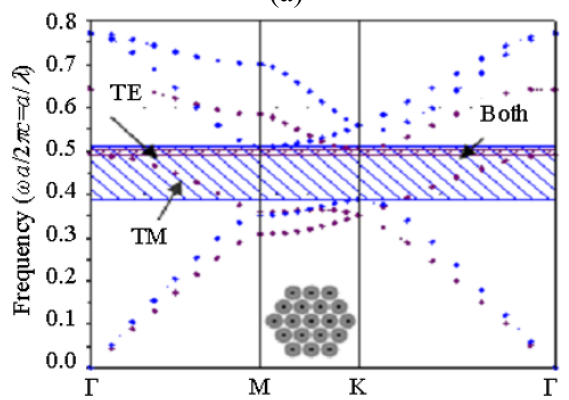

(b)

Fig. 6 Photonic band structure for TE and TM polarization modes in the $2 \mathrm{D}$ hexagonal lattice of $\mathrm{ZnS}$ rods covered with the air shell layer for (a) $t_{s}=0.46 a$ and (b) $t_{s}=0.39 a$ 
the inner rod radius $r_{c}=0.01 a$, the outer radius $r_{s}=0.47 a$ and the shell thickness $t_{s}=0.46 a$, for which the CPBG achieves its maximum relative width of $\Delta w / w_{\mathrm{g}}=6.9 \%$, where $\Delta w$ and $w_{\mathrm{g}}$ are the frequency width and the middle frequency of the gap, respectively. No we consider the internal radius $r_{c}=0.08 a, \quad r_{s}=0.47 a$ and $t_{s}=0.39 a$.

Figure 6(b) shows the dispersion curves for this case. The width of the CPBG is $\Delta w / w_{\mathrm{g}}=2 \%$ and decreases brusquely because of the reduced air filling factor. Figure 7 indicates the photonic gap map of a 2D hexagonal lattice of $\mathrm{ZnS}$ rods covered with the air shell layer, where the spectral widths are plotted against the rod radius $r_{c} / a$. The CPBG disappears completely when the rods are not covered by air layers.

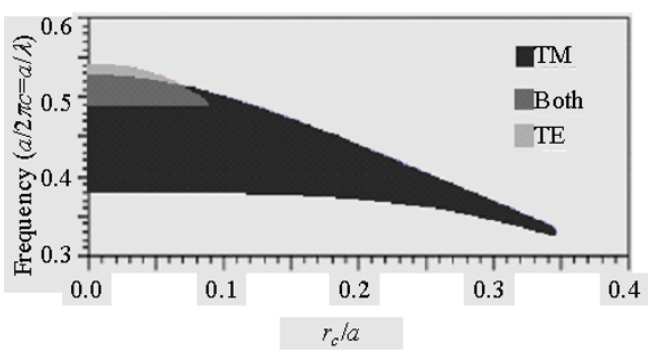

Fig. 7 Photonic gap map for the hexagonal lattice of $\mathrm{ZnS}$ rods covered with the air shell layer in the $\mathrm{TiO}_{2}$ background $\left(r_{s}=0.47 a\right)$.

The critical value of $t_{s}$, for which the width of the CPBG appears, is $0.384 a\left(r_{c} / a=0.086\right)$. For $t_{s}$ greater than $0.384 a$, the width of the CPBG increases. The largest width of the CPBG can be obtained around $r_{c} / a=0.01 \quad\left(t_{s}=0.46 a\right)$. Hence, it seems that the full PBG width is strongly affected by the thickness of the interfacial layer. The result demonstrates that the CPBG is enlarged when the $\mathrm{ZnS}$ rods are covered by the air shell layer.

\subsection{Square lattice}

In this section the effect of the thickness of the shell layer on the CPBG for the square lattice of $\mathrm{ZnS}$ rods surrounded by the air as shown in Fig. 5(b) is investigated. The structural parameters are the same as in Section 4.1. The CPBG is opened up from the overlap between of $H_{2-3}$ and $E_{3-4}$ gaps. As shown in Figs. 8(a) and 8(b), the square arrangement of $\mathrm{ZnS}$ rods covered with the air shell layer exhibits a very narrow CPBG. Figure 8(a) represents the band gap structure for $r_{c}=0.02 a, r_{s}=0.47 a$ and $t_{s}=0.45 a$. Only one tiny $\mathrm{CPBG}$ exists and achieves its greatest relative width of $\Delta w / w_{\mathrm{g}}=1.28 \%$.

Now consider the case when $r_{c}$ is $0.044 a$ and $t_{s}$ is $0.426 a$, the relative width of the CPBG is $\Delta w / w_{\mathrm{g}}=0.8 \%$. Figure 8 (b) clearly illustrates that the width is reduced from that in Fig. 8(a). The photonic gap map of a $2 \mathrm{D}$ square lattice of $\mathrm{ZnS}$ rods covered with the air shell layer is shown in Fig. 9. The width of the CPBG appears for $r_{c}=0.058 a$ and $t_{s}=0.412 a$,

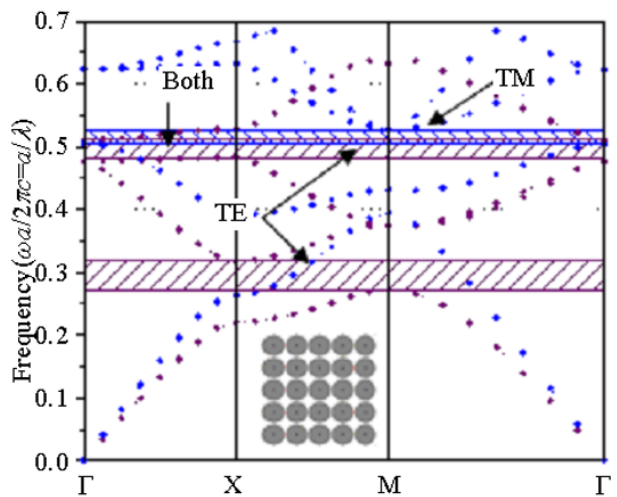

(a)

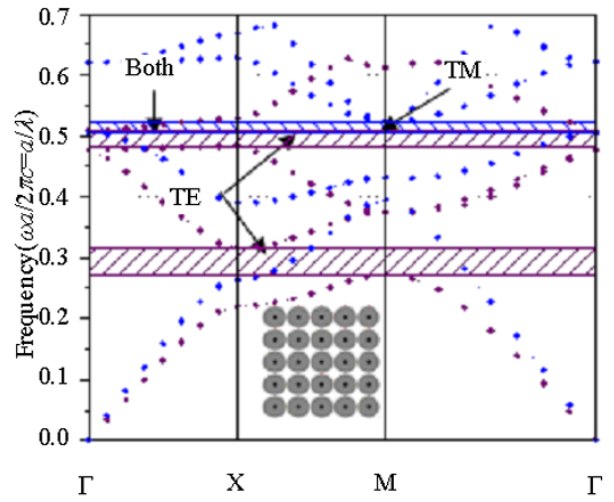

(b)

Fig. 8 Photonic band structure for TE and TM polarization modes in the 2D square lattice of $\mathrm{ZnS}$ rods covered with the air shell layer for (a) $t_{s}=0.45 a$ and (b) $t_{s}=0.426 a$.

and the overlap between $H_{2-3}$ and $E_{3-4}$ gaps diminishes when $r_{c}$ increases, and at large values of $r_{c}$, the width of the CPBG narrows down and then closes when $r_{c}$ goes beyond $0.058 a\left(t_{s}\right.$ is lower than 
$0.412 a$ ). The photonic band-structure calculations demonstrate that the width of the CPBG is governed by the thickness of the shell layer.

The presence of an interfacial air layer surrounding the $\mathrm{ZnS}$ rods has an obvious influence on the width of the CPBG for the square lattice structure. The relative width of the CPBG increases with an increase in the thickness of the shell layer. Therefore, in order to obtain the largest CPBG, we should suitably choose the thickness of the air shell layer.

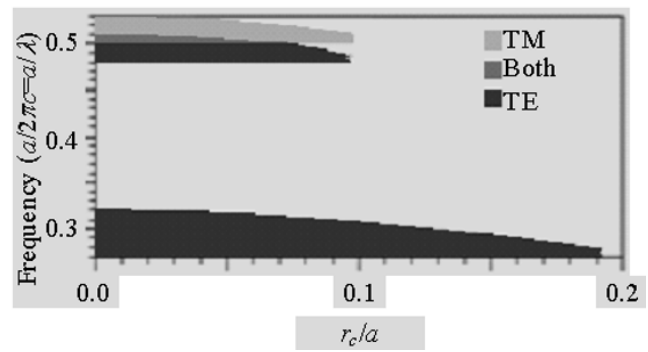

Fig. 9 Photonic gap map for the square lattice of $\mathrm{ZnS}$ rods covered with the air shell layer in the $\mathrm{TiO}_{2}$ background $\left(r_{s}=\right.$ $0.47 a)$.

\section{Conclusions}

We have theoretically demonstrated the possibilities of the adjustment of optical properties of the $\mathrm{TiO}_{2}$ matrix in the presence of $\mathrm{ZnS}$ nano-objects. The changes in the refractive index allows us to obtain a new composite material which can have adjustable PBGs. The effect of $\mathrm{ZnS}$ nanoparticles in the host matrix on the PBG structure is investigated. Our results imply that the CPBG can be enlarged by adding $\mathrm{ZnS}$ inclusions in the background matrix. Complete photonic band gaps in 2D hexagonal and square lattices considering an interfacial layer between the $\mathrm{ZnS}$ rods and $\mathrm{TiO}_{2}$ matrix have been studied. Photonic band-structure calculations reveal a strong effect of the thickness of this interfacial layer on complete photonic gaps. If the thickness of the shell layer decreases until it reaches a certain threshold value, the CPBG begins to narrow down until it disappears completely. This implies that the existence of the air shell layer leads to larger complete photonic gaps in the two cases (hexagonal and square lattices).

We believe that the present results are significant to increase the possibilities for experimentalists to realize a larger CPBG.

Open Access This article is distributed under the terms of the Creative Commons Attribution License which permits any use, distribution, and reproduction in any medium, provided the original author(s) and source are credited.

\section{References}

[1] E. Yablonovitch, "Inhibited spontaneous emission in solid-state physics and electronics," Phys. Rev Lett., vol. 58, no. 20, pp. 2059-2061, 1987.

[2] S. John, "Strong localization of photons in certain disordered dielectric superlattices," Phys. Rev. Lett., vol. 58, no. 23, pp. 2486-2489, 1987.

[3] J. D. Joannopoulos, S. G. Johnson, J. N. Winn, and R. D. Meade, Photonic Crystals: Molding the Flow of Light. Princeton: Princeton University Press, 2008.

[4] K. Sakoda, Optical Properties of Photonic Crystals. Germany: Springer, 2000.

[5] P. Chrysicopoulou, D. Davazoglou, Chr. Trapalis, and G. Kordas, "Optical properties of very thin $(<100 \mathrm{~nm})$ sol-gel $\mathrm{TiO}_{2}$ films," Thin Solid Films, vol. 323, no. 1-2, pp. 188-193, 1998.

[6] C. Falcony, M. Garcia, A. Ortiz, and J. C. Alonso "Luminescent properties of $\mathrm{ZnS}$ : Mn films deposited by spray pyrolysis," J. Appl. Phys., vol. 72, no. 4, pp. 1525-1527, 1992.

[7] S. Yanagida, H. Kawakami, Y. Midori, H. Kizumoto, C. Pac, and Y. Wada, "Semiconductor photocatalysis. ZnS-nanocrystallite-catalyzed photooxidation of organic compounds," Bull. Chem. Soc., vol. 68, no. 7, pp. 1811-1823, 1995 .

[8] W. Park, J. S. King, C. W. Neff, C. Liddell, and C. J. Summers, "ZnS-based photonic crystals," Phys. Stat. Sol., vol. 229, no. 2, pp. 949-960, 2002.

[9] T. Trifonov, L. F. Marsal, A. Rodríguez, J. Pallarès, 
and R. Alcubilla, "Analysis of photonic band gaps in two- dimensional photonic crystals with rods covered by a thin interfacial layer," Phys. Rev. B., vol. 70, no. 19, pp. 195108-1-195108-8, 2004.

[10] H. Xiao, D. Z. Yao, and C. X. Wang, "Analysis of the peculiar shift of the low-frequency end of a variable photonic band gap," Appl. Phys. B, vol. 87, no. 3, pp. 463-467, 2007.

[11] V. Babin, P. Garstecki, and R. Hołyst, "Multiple photonic band gaps in the structures composed of core-shell particles," J. Appl. Phys., vol. 94, no. 7, pp. 4244-4247, 2003.

[12] T. Pan, F. Zhuang, and Z. Y. Li, "Absolute photonic band gaps in a two-dimensional photonic crystal with hollow anisotropic rods," Solid State Commun., vol. 129, no. 8, pp. 501-506, 2004.
[13] J. C. Maxwell-Garnett, "Colors in metal glasses and in metallic films," Phil. Trans. R. Soc. A, vol. 203, no. 359-371, pp. 385-420, 1904.

[14] C. F. Bohren and D. R. Huffman, Absorption and scattering of light by small particles. New York: Wiley-VCH, 2004.

[15] M. H. Kok, et al., "Photonic band gap effect and structural color from silver nanoparticle gelatin emulsion," Phys. Rev. E, vol. 72, no. 4, pp. 047601-1-047601-4, 2005.

[16] E. D. Palik, Handbook of Optical Constants of Solids. New York: Academic Press, 1985.

[17] A. Taflov and S. C. Hagness, Computational Electro-dynamics:the finite-difference time- domain Method, 2 edition. Boston: Artech House Publishers, 2000. 